\title{
Defining aggressive or early progressing nononcogene-addicted non-small-cell lung cancer: a separate disease entity?
}

\author{
Martin Reck ${ }^{1}$, Keith M Kerr², Christian Grohé ${ }^{3}$, Christian Manegold ${ }^{4}$, Nick Pavlakis ${ }^{5}$, Luis \\ Paz-Ares ${ }^{6}$, Rudolf M Huber ${ }^{7}$, Sanjay Popat ${ }^{8}$, Nick Thatcher ${ }^{9}$, Keunchil Park ${ }^{10}$, Frank \\ Hilberg ${ }^{11}$, José Barrueco ${ }^{12}$ \& Rolf Kaiser*,13 \\ ${ }^{1}$ Department of Thoracic Oncology, Lung Clinic Grosshansdorf, Member of the German Center for Lung Research (DZL), \\ Grosshansdorf, Germany \\ ${ }^{2}$ Department of Pathology, Aberdeen University Medical School, Aberdeen Royal Infirmary, Aberdeen, Scotland \\ ${ }^{3}$ Department of Respiratory Diseases, Evangelische Lungenklinik Berlin, Lindenberger Weg 27, Berlin, Germany \\ ${ }^{4}$ Medical Faculty Mannheim, University of Heidelberg, Theodor-Kutzer-Ufer 1-3, 68167 Mannheim, Germany \\ ${ }^{5}$ Department of Medical Oncology, Royal North Shore Hospital (Sydney University), Reserve Road, St Leonards 2065, New South \\ Wales, Australia \\ ${ }^{6}$ Medical Oncology Department, University Hospital 12 de Octubre, Complutense University, CNIO \& CiberOnc, Madrid, Spain \\ ${ }^{7}$ Division of Respiratory Medicine \& Thoracic Oncology, Ludwig Maximilians University of Munich, \& Thoracic Oncology Centre \\ Munich, Member of the German Center for Lung Research (DZL CPC-M) Munich, Germany \\ ${ }^{8}$ Department of Medicine, Royal Marsden Hospital NHS Foundation Trust, London, UK \\ ${ }^{9}$ Department of Medical Oncology, Christie Hospital NHS Trust, Wilmslow Road, Manchester, UK \\ ${ }^{10}$ Department of Medicine, Samsung Medical Center, Sungkyunkwan University School of Medicine, Seoul, Korea \\ ${ }^{11}$ Boehringer Ingelheim RCV GmbH \& Co. KG, A-1121, Vienna, Austria \\ ${ }^{12}$ Boehringer Ingelheim Pharmaceuticals Inc., Ridgefield, Connecticut, CT 06877, USA \\ ${ }^{13}$ Boehringer Ingelheim Pharma GmbH \& Co, KG, Germany \& Institute of Pharmacology, Johannes Gutenberg-University Mainz, \\ Germany \\ *Author for correspondence: rolf.kaiser@boehringer-ingelheim.com
}

A substantial proportion of patients with nononcogene-addicted non-small-cell lung cancer (NSCLC) has 'aggressive disease', as reflected in short time to progression or lack of disease control with initial platinum-based chemotherapy. Recently, clinical correlates of aggressive disease behavior during first-line therapy have been shown to predict greater benefit from addition of nintedanib to second-line docetaxel in adenocarcinoma NSCLC. Positive predictive effects of aggressive disease have since been reported with other anti-angiogenic agents (ramucirumab and bevacizumab), while such features may negatively impact on outcomes with nivolumab in nonsquamous NSCLC with low PD-L1 expression. Based on a review of the clinical data, we recommend aggressive nonsquamous NSCLC should be defined by progression within $<6-9$ months of first-line treatment initiation.

Lay abstract: Chemotherapy with platinum-containing drugs was for many years the standard initial treatment for advanced NSCLC. Today's treatment options also include drugs that help the immune system to attack the cancer ('immunotherapies'). These drugs may be used with or without chemotherapy, depending on the molecular profile of the tumor. However, many patients have a fast-growing ('aggressive') form of NSCLC and so gain little benefit from initial chemotherapy. This patient subgroup has often been overlooked in clinical research to date. In this article, we discuss how 'aggressive NSCLC' can be defined and consider how many patients are affected. We also look at the impact of aggressive disease on the effectiveness of other treatments used after initial chemotherapy. Based on a thorough review of the evidence, we recommend that patients who need to change treatment within 6-9 months of starting initial therapy should be defined as having aggressive NSCLC. We hope this definition can help doctors to recognize aggressive tumors and adapt treatment accordingly to improve patients' outcome.

First draft submitted: 18 December 2018; Accepted for publication: 28 January 2019; Published online: 13 February 2019

Keywords: aggressive • anti-angiogenic therapy • non-small-cell lung cancer 
Enormous strides have been made in the management of advanced NSCLC over the past decades [1]. Insights into the biology and genetics of the disease have yielded novel therapeutic strategies and, in a subset of patients, enable individualized treatment targeting the oncogenic mutations that drive tumor growth [1-3]. Nevertheless, more than three quarters of advanced NSCLCs lack an 'actionable' driver mutation for which a specific targeted therapy is approved [3]. For many years, the mainstay of first-line therapy for this population was chemotherapy with a platinum compound (cisplatin or carboplatin) in combination with a third-generation cytotoxic agent, such as an antitubulin therapy (docetaxel, paclitaxel, nab-paclitaxel or vinorelbine) or antimetabolite (pemetrexed or gemcitabine) [4-7]. These regimens may be combined with the anti-VEGF antibody bevacizumab for nonsquamous tumors $[4,8,9]$. For squamous tumors, the EGFR inhibitor necitumumab is approved for use with gemcitabine/cisplatin $[4,9]$. In biomarker-unselected populations, first-line platinum-based doublets provide an overall response rate (ORR) of 20-30\% and median time-to-progression (TTP) of 3.5-5.5 months [5].

First-line treatment of nononcogene-addicted NSCLC is currently undergoing a significant shift with the introduction of immunotherapies that reactivate antitumor immunity through inhibition of the PD-1/PD-L1 axis [10-13]. Only the PD-1 inhibitor pembrolizumab is currently approved for first-line use, either as monotherapy in patients with PD-L1 tumor proportion score (TPS) $\geq 50 \%$ ( $~ 30 \%$ of nononcogene-addicted NSCLC) or in combination with chemotherapy for nonsquamous tumors irrespective of PD-L1 expression [10,14]. The first of these indications was approved following demonstration of significant overall survival (OS) benefit with pembrolizumab versus chemotherapy in patients with PD-L1 TPS $\geq 50 \%$ in the Phase III KEYNOTE-024 trial [10,14]. The antiPD-1 agent nivolumab, in contrast, failed to demonstrate efficacy as monotherapy when compared with first-line chemotherapy in a Phase III trial in patients with PD-L1 TPS $\geq 5 \%$ [15].

More recently, both pembrolizumab and the PD-L1 inhibitor atezolizumab have shown significant benefits when added to first-line chemotherapy ( \pm bevacizumab) in Phase III trials in nonsquamous and squamous NSCLC, irrespective of PD-L1 expression level $[11,12,16,17]$. Nivolumab has also demonstrated efficacy in the first-line setting when combined with a second immunotherapy, the CTLA-4 blocker ipilimumab, in PD-L1-unselected NSCLC with high tumor mutational burden [13]. A secondary end point of the same trial showed progression-free survival (PFS) and response increases with nivolumab plus chemotherapy versus chemotherapy alone in PD-L1-negative NSCLC regardless of histology or tumor mutational burden [18]. Currently, only pembrolizumab is approved for use in combination with first-line chemotherapy (for nonsquamous NSCLC) [14], but recent results, guidelines [4] and potential future regulatory approvals will likely stimulate increasing incorporation of immunotherapies, with or without chemotherapy, into initial treatment. These tremendous advances raise significant questions for subsequent management, since there is little evidence regarding the efficacy of current second-line options after initial immunotherapy.

First-line chemotherapy provides disease control for most patients with nononcogene-addicted advanced NSCLC, but almost all will eventually develop progressive disease (PD) $[11,15,19]$. Although several second-line therapies are approved, only $\sim 50-60 \%$ of patients in clinical trials go on to receive further therapy and even fewer may receive second-line treatment in real-world settings $[11,15,20,21]$. In a German cohort of 804 patients with nononcogeneaddicted nonsquamous NSCLC, only $37 \%$ of patients treated with first-line therapy received a second-line regimen (C Grohé, Unpublished Observations). Low utilization of second-line therapy likely reflects rapidly declining clinical condition in a substantial number of patients [22].

Depending on first-line therapy, tumor histology and PD-L1 expression, second-line options can include antiangiogenic therapies (nintedanib or ramucirumab) in combination with docetaxel, single-agent immunotherapies (pembrolizumab, nivolumab or atezolizumab), cytotoxic monotherapy with docetaxel or pemetrexed, the ErbB family blocker afatinib, or the EGFR inhibitor erlotinib (EGFR wild-type tumors; Figure 1) [4]. With a range of second-line therapies now approved, there is significant interest in the development of predictive factors to support clinical decision making. Against this background, systematic analyses of Phase III data for nintedanib for the first time showed that clinical correlates of 'aggressive' disease behavior in the first-line setting had predictive relevance for the efficacy of subsequent therapy [23]. These observations have since been extended in analyses of other second-line therapies [24-27].

Given the emerging importance of these clinical markers, this narrative review summarizes current knowledge regarding aggressive disease in patients with nononcogene-addicted advanced NSCLC. We discuss the concept, definition, prevalence and clinical features of these tumors, consider the pathophysiological mechanisms that may 


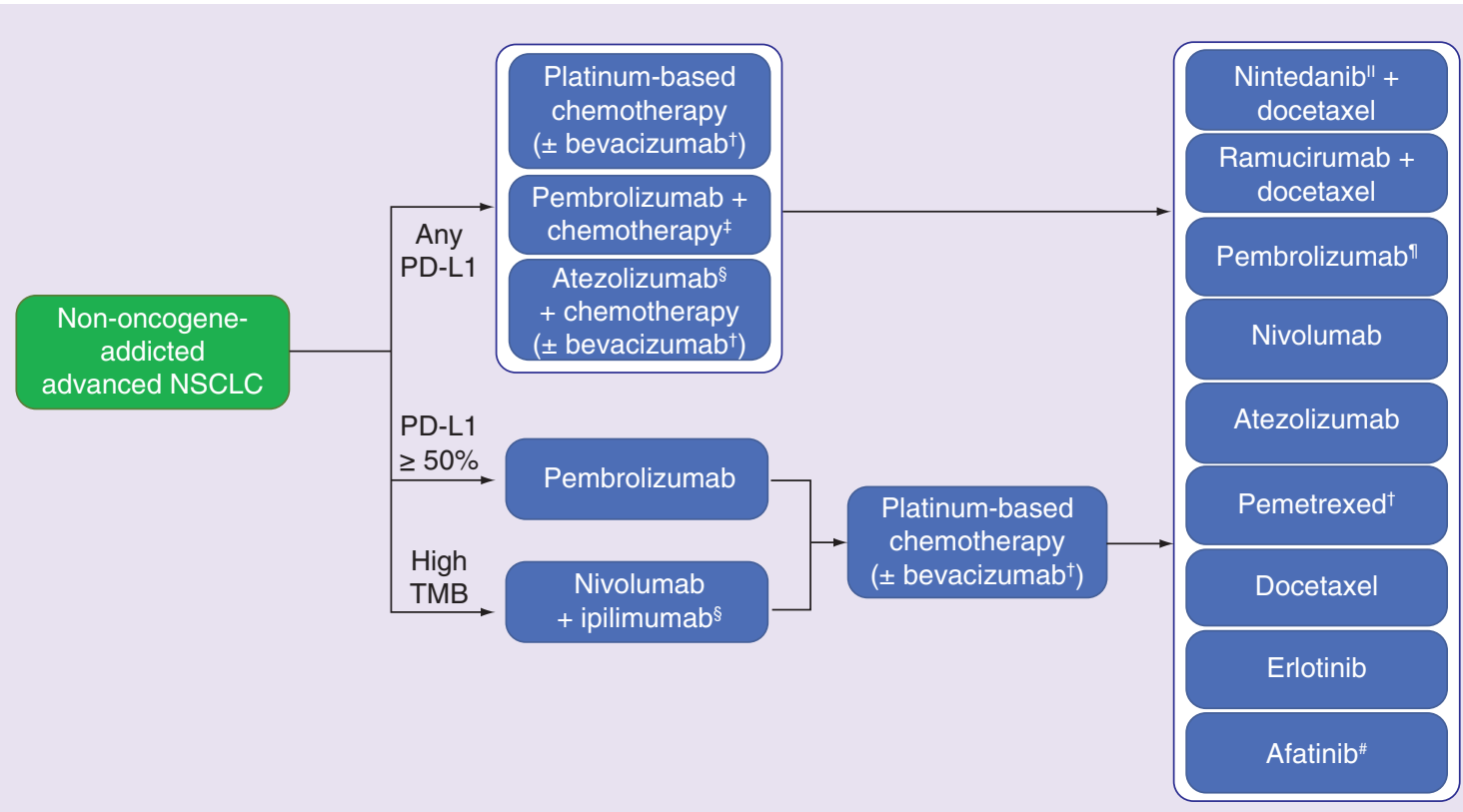

Figure 1. Treatment options for patients with nononcogene-addicted non-small-cell lung cancer.

†Tumors with nonsquamous histology.

$\ddagger$ Only approved for tumors with nonsquamous histology at the time of writing.

$\S$ Indication not approved at the time of writing.

"Tumors with adenocarcinoma histology.

I Tumors with PD-L1 expression $>1 \%$.

\#Tumors with squamous histology.

NSCLC: Nononcogene-addicted non-small-cell lung cancer; TMB: Tumor mutational burden.

Adapted with permission from [4].

underlie aggressive disease behavior, and explore how current clinical data may redefine the treatment landscape for these tumors. Finally, we propose a clinical definition of aggressive disease to help harmonize future research that aims to improve the identification and management of this under-recognized, and hitherto neglected, patient population.

\section{Concept of aggressive NSCLC}

Every physician treating patients with cancer will have experience of tumors that progress rapidly and are not amenable to treatment. Due to their short survival times and expectation of treatment refractoriness, these patients may be under-represented in clinical trials and have never been a focus of intensive research. Moreover, the concept of aggressive disease remains poorly defined and rarely considered in either preclinical/clinical research or clinical practice.

In cancer biology, the term 'aggressive' is broadly used to describe tumors that develop, progress or metastasize more quickly [28]. Aggressive disease behavior is, therefore, expected to manifest in poor clinical outcomes, such as nonresponse, lack of disease control, more rapid TTP and shorter duration of survival. Although associated with unfavorable outcomes, aggressive disease is not synonymous with poor prognosis per se, as it implies the presence of a pernicious underlying pathophysiology and excludes patient-related poor prognostic factors, such as age and performance status (PS) [29]. Clinically, the concept of 'aggressive disease' may be useful for identifying a subset of patients with unfavorable clinical course of disease (prognostic relevance) and/or who may derive particular benefit from specific therapeutic approaches (predictive relevance).

Analyses to date have utilized operational definitions of aggressive NSCLC to capture early progression on or after platinum-containing chemotherapy, based on either the timing of second-line therapy or best response attained during first-line treatment. The former, time-based measures have indexed the second-line baseline either to the start of first-line therapy (time since start of first-line therapy [TSFLT]) or to the end of first-line therapy (time since end of first-line therapy; TEFLT), the key difference between these variables being the exclusion of 
the on-treatment period from TEFLT [30]. TSFLT and TEFLT are analogous to the progression-free interval and platinum-free interval, respectively, which are routinely applied in the classification of small cell lung cancer (SCLC) and ovarian cancer [31,32]. In NSCLC, TSFLT cut-points of $<3$ months, $\leq 5$ months and $<9$ months have been explored in clinical datasets $[25,30,33]$. The TSFLT cut-point of $<9$ months was selected as a potentially optimal threshold in prognostic and predictive analyses of two Phase III studies of nintedanib [23], while TSFLT $\leq 5$ months was based on median PFS of $\sim 5$ months reported in interventional and observational NSCLC studies with first-line platinum-based chemotherapy [30,34,35]. For TEFLT, cut-points of $<3$ and $\leq 6$ months have been evaluated, which correspond to definitions of platinum resistance used in SCLC and ovarian cancer, respectively [30-32].

Aggressive disease has also been defined by lack of disease control during initial treatment, as indicated by PD as best response to first-line therapy (PD-FLT) $[25,30,33]$. Compared with TSFLT-based definitions, PD-FLT is a more restrictive categorization because it excludes patients who attain even short-lived responses. These classifications bear some relation to the definitions of primary (intrinsic) and secondary (acquired) resistance that are used in EGFR inhibitor-treated NSCLC [36]. Whereas PD-FLT is similar to primary resistance (although primary resistance may or may not include patients with stable disease [SD]), TSFLT-defined aggressive disease can also include tumors with secondary resistance depending when resistance emerges [36]. Indeed a key limitation of both TSFLT and PD-FLT definitions is their inability to distinguish between de novo aggressive disease and treatment resistance. It is worth noting that 'refractory disease' has been used variously to refer to PD-FLT, TSFLT $\leq 5$ months and TEFLT $<3$ months $[25,30,37]$. In this review, we will favor the TSFLT, TEFLT and PD-FLT descriptors throughout to specify the definition being applied in each case.

The term 'hyperprogressive disease' (HPD) has been recently coined to describe a new pattern of nonresponse in which tumors exhibit a paradoxical acceleration of growth during treatment with immunotherapies [38]. Different investigators have defined HPD by a $>50 \%$ or $\geq$ twofold increase in tumor growth rate following immunotherapy initiation, relative to the pretreatment period [38,39]. HPD is distinguished from usual PD in that it is dependent on treatment and represents a marked worsening of disease, rather than simple nonefficacy [38]. As a treatment-related phenomenon, HPD is distinct from the aggressive disease under discussion here.

In contrast to HPD, pseudoprogression is a transient lesion enlargement resulting from tumor infiltration with immune cells during treatment with immunotherapy [38]. As first-line use of immunotherapies increases, it will be important to avoid misattributing true $\mathrm{PD}$ as pseudoprogression, which could otherwise reduce patient access to second-line therapies.

\section{Prevalence of aggressive NSCLC}

There is consistent evidence that a significant subpopulation of patients with nononcogene-addicted advanced NSCLC derive little or no benefit from first-line chemotherapy. An early randomized study comparing four platinum-based doublets reported a high rate of PD-FLT of 40-49\%, with a correspondingly short median TTP of 3.1-4.2 months across the study arms [40]. Other trials of platinum combinations from the same era observed a lower PD-FLT rate of $17-28 \%$ in patients unselected according to histology or biomarker expression [41-43]. More recent estimates of the PD-FLT rate with chemotherapy are provided by the control arms of first-line immunotherapy trials, which excluded patients with nonsquamous tumors and EGFR mutation/anaplastic lymphoma kinase (ALK) rearrangement or required these patients to have received molecularly targeted therapy $[11,12,15,16]$. In these studies, PD-FLT rates were reported at $17 \%$ with chemotherapy alone and $27 \%$ with chemotherapy plus bevacizumab in nonsquamous populations, $14 \%$ with chemotherapy in squamous NSCLC, and 10\% with chemotherapy alone in patients with any histology and PD-L1 TPS $\geq 5 \%[11,12,15,16]$.

It is more challenging to extract time-based estimates of aggressive disease from these studies, since landmark analyses of TTP or PFS at early time points have rarely been reported. TTP/PFS curves suggest that around 20$30 \%$ of patients progress and/or die within 3 months of chemotherapy initiation [34,41]. Consistent with this, PFS rates were estimated at 73 and $37 \%$ at 3 and 6 months, respectively, with cisplatin plus gemcitabine in a Phase III study in squamous NSCLC [44]. A 6-month PFS rate of 50\% was reported with chemotherapy alone in $E G F R / A L K$ wild-type NSCLC of any histology and PD-L1 TPS $>50 \%$ in KEYNOTE-024 [10]. Prospective estimates of aggressive disease prevalence are also provided by maintenance therapy trials in which response assessments after four cycles of first-line treatment are used to determine eligibility for treatment de-escalation (Table 1). In these studies, $14-31 \%$ of patients experienced progression after four cycles of induction therapy with platinum-based doublets administered over 3-4 months [45-51]. 


\begin{tabular}{|c|c|c|c|c|c|}
\hline Trial & Histology & $\mathrm{n}$ & Induction regimen (no. cycles, cycle duration) & PD rate at end of induction & Ref. \\
\hline IFCT-GFPC 0502 & Any & 834 & Cisplatin + gemcitabine (four cycles, Q3W) & $191 / 834(23 \%)$ & [45] \\
\hline AVAPERL & Nonsquamous & 376 & $\begin{array}{l}\text { Cisplatin + pemetrexed + bevacizumab (four cycles, } \\
\text { Q3W) }\end{array}$ & $52 / 376(14 \%)$ & [46] \\
\hline Fidias 2008 & Any & 566 & Carboplatin + gemcitabine (four cycles, Q3W) & $147 / 566(26 \%)$ & [47] \\
\hline PARAMOUNT & Nonsquamous & 939 & Cisplatin + pemetrexed (four cycles, Q3W) & $217 / 939(23 \%)$ & [48] \\
\hline TITAN/SATURN & Any & 1949 & Platinum-based doublets (four cycles, Q3W) & $424 / 1949(22 \%)$ & [49] \\
\hline Belani 2003 & Any & 401 & $\begin{array}{l}\text { Carboplatin + paclitaxel (four cycles, Q4W or two } \\
\text { cycles, Q8W) }\end{array}$ & NR/NR (31\%) & [50] \\
\hline
\end{tabular}

NR: Not reported; PD: Progressive disease; Q3W: Every 3 weeks; Q4W: Every 4 weeks; Q8W: Every 8 weeks.

Relevant data are also available from second-/third-line studies in which outcomes of prior therapy are retrospectively assessed at enrollment and reported in baseline characteristics (Table 2). Although these patients represent a more selected group (i.e., those for whom second-line therapy is considered suitable), they are the clinically relevant population when considering the potential predictive relevance of first-line outcomes for subsequent treatment strategies. Trials enrolling patients with any histological subtype who had previously received a single line of chemotherapy have found prior PD-FLT rates of $20-30 \%$ in their respective populations $[24,33,56,57,61,63]$. In second-line nonsquamous NSCLC, the rate of PD-FLT was $18 \%$ in the adenocarcinoma subgroup of LUMELung 1 and 39\% in CheckMate 057 [30,54]. TSFLT was $<6$ months in $25-35 \%$ of patients and $<9$ months in $43-62 \%$ of patients across the studies shown in Table 2 [24-26,30,33,37,60,62]. Approximately $70 \%$ of patients in these studies had TEFLT $\leq 6$ months [30,57]. As would be expected, in the adenocarcinoma population of LUME-Lung 1 , the subgroup with TSFLT $<9$ months included almost all the patients with PD-FLT, but around two-thirds of patients with TSFLT $<9$ months had attained at least disease stabilization during first-line chemotherapy [30].

Observational data suggest that similar numbers of patients fail to achieve disease control with initial chemotherapy in less selected populations treated with a broader range of regimens [65-67]. In a German cohort of 804 patients with nonsquamous tumors, $48 \%$ discontinued first-line chemotherapy after just 1-3 cycles (C GrOHÉ, UnPublished ObSERVATIONS).

The data above refer to first-line treatment with conventional chemotherapy regimens. Available data with immunotherapies in this setting, alone or in combination with chemotherapy, are limited at present. In firstline Phase III trials, PD-FLT rates with pembrolizumab plus chemotherapy versus chemotherapy alone were 9 versus $18 \%$ in nonsquamous NSCLC and 6 versus $14 \%$ in squamous tumors [11,16]. Results from ImPower 150 indicate an $18 \% \mathrm{PD}$ rate with atezolizumab in combination with bevacizumab plus chemotherapy in nonsquamous $E G F R / A L K$ wild-type NSCLC, compared with $27 \%$ in the control arm [12]. In the Phase III CheckMate 026 trial, the rate of PD-FLT was numerically higher with nivolumab monotherapy $(27 \%)$ than with chemotherapy alone $(10 \%)$ in patients with PD-L1 TPS $\geq 5 \%$ [15].

\section{Prognosis of aggressive NSCLC}

One of the first prospective datasets to associate aggressive disease during first-line therapy with outcomes in subsequent lines was the randomized Phase III JMEI trial of second-line pemetrexed versus docetaxel [56,57]. In a secondary analysis of both arms, patients with prior PD-FLT had significantly shorter median OS (4.6 months) than patients with prior SD (10.5 months) or ORR (15.8 months) in univariate and multivariate analyses (both $\mathrm{p}<0.001)$ [57]. Median OS was also shorter in patients with TEFLT $\leq 3$ months (6.9 months) than in patients with TEFLT 3-6 months (9.2 months) or TEFLT $\geq 6$ months (9.3 months; univariate $\mathrm{p}=0.001$ ), although this was not significant after adjustment $(\mathrm{p}=0.183)$ [57].

Subsequent studies have also highlighted a negative prognostic impact of aggressive disease behavior in first-line treatment. In trial BR.21, which randomized patients ineligible for further chemotherapy to erlotinib or placebo as second- or later-line therapy, both PD-FLT and time since diagnosis of $<12$ months were independently and significantly correlated with shorter OS ( $\mathrm{p}=0.022$ and $\mathrm{p}=0.012$, respectively) in the erlotinib arm [68]. Nonresponse to prior chemotherapy was associated with a $25 \%$ increase in the risk of death in a meta-analysis of nine trials of second-line chemotherapy, although the authors did not investigate PD-FLT specifically [69]. Poor outcomes have been reported in second-line trials that selected a population with aggressive disease based on PD during or immediately following induction therapy or TEFLT $<3$ months [37,49,67]. More recently, analyses of the control arm 


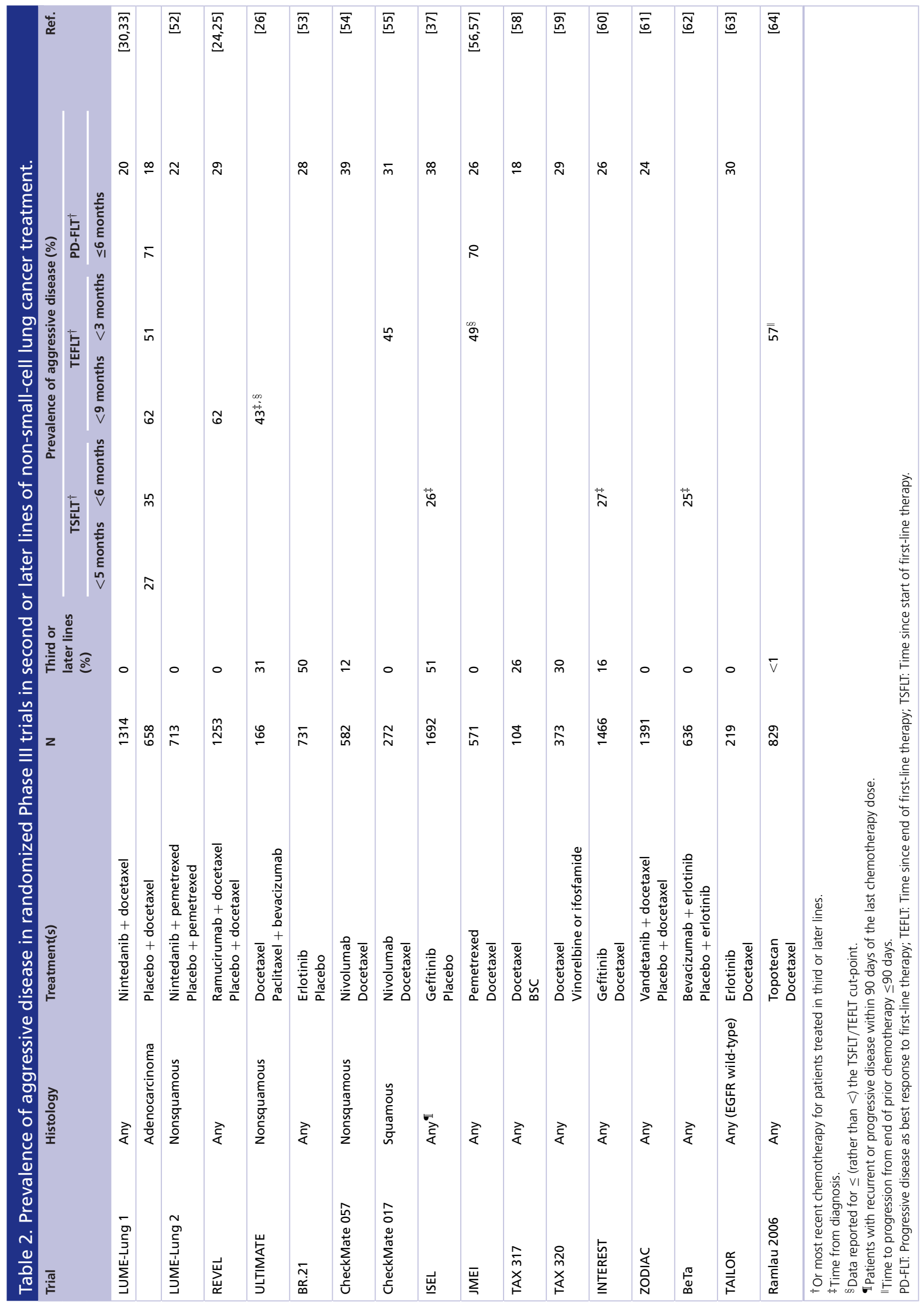


of LUME-Lung 2 identified TSFLT as potentially prognostic for both investigator-assessed and centrally assessed PFS in patients receiving second-line pemetrexed [23].

Observational data support a prognostic impact of first-line outcomes on subsequent lines of therapy in real-world settings $[66,67,70]$. For example, in a German nonsquamous cohort, earlier discontinuation of first-line chemotherapy (after 1-3 cycles) was associated with both lower use and shorter duration of second-line treatment, as compared with patients who had received 4-6 cycles of first-line therapy (C Grohé, Unpublished ObSERvations). Another retrospective German study demonstrated a significant negative prognostic impact on OS of both PD-FLT and TSFLT $<9$ months, with the latter also being shown for the adenocarcinoma population [67].

\section{Demographic \& clinical characteristics of patients with aggressive NSCLC}

Few correlations between aggressive disease and demographic or clinical variables have been reported. Compared with the overall populations, the TSFLT $<9$ months subgroup of LUME-Lung 1 and the PD-FLT subgroup of REVEL had generally comparable baseline characteristics, although female patients may have been slightly underrepresented in the REVEL PD-FLT subgroup $[24,25,30]$. In a German observational study, failure to achieve disease control with first-line treatment was associated with the presence of adrenal metastases, as well as the patient-related poor prognostic factors of PS and self-reported weight loss at diagnosis [66].

\section{Predictive relevance of aggressive NSCLC for efficacy of targeted therapies in second \& subsequent lines}

Nintedanib

Nintedanib is an oral, small-molecule, triple angiokinase inhibitor of VEGFR 1-3, FGF receptor 1-3 and PDGF receptor $\alpha / \beta$, along with FLT3 and SRC family kinases [71]. It is approved in the EU for use in combination with docetaxel for the second-line treatment of adenocarcinoma NSCLC based on the results of the Phase III LUME-Lung 1 trial [33]. In that study, the addition of nintedanib to docetaxel significantly improved the primary end point of PFS, compared with placebo plus docetaxel, in the overall population of 1314 patients previously treated with a single line of chemotherapy (hazard ratio [HR]: 0.79; 95\% CI: $0.68-0.92 ; \mathrm{p}=0.0019$ ). A prespecified key secondary end point analysis demonstrated a significant increase in OS favoring the nintedanib arm among patients with adenocarcinoma histology (HR: 0.83; 95\% CI: 0.70-0.99; $\mathrm{p}=0.0359$ ).

The nintedanib clinical development program in NSCLC provided the first evidence that prior aggressive tumor behavior influenced the efficacy of a targeted therapy [23]. To identify potential clinical markers of nintedanib treatment effect, the investigators undertook a systematic evaluation of data from LUME-Lung 1 along with a second Phase III trial, LUME-Lung 2, which evaluated second-line nintedanib plus pemetrexed versus placebo plus pemetrexed in advanced NSCLC [23,52]. In a hypothesis-generating stepwise selection and recursive partitioning analyses of PFS data from LUME-Lung 2, TSFLT was significantly associated with prognosis in the placebo arm and was the only prognostic variable also showing significant predictive relevance for PFS. HR by treatment interaction plots suggested that a TSFLT cut-point of approximately 9 months provided an optimal binary split for the predictive effect. Initial validation of the predictive relevance of TSFLT $<9$ months was provided by OS data from LUME-Lung 2 and by centrally assessed PFS from LUME-Lung 1. In patients with adenocarcinoma and TSFLT $<9$ months, median PFS in LUME-Lung 1 was 3.6 months in the nintedanib arm versus 1.5 months with chemotherapy alone in the primary analysis (HR: 0.63; 95\% CI: 0.48-0.83; $\mathrm{p}=0.0008$ ).

The predefined stepwise hierarchical testing of the key secondary end point of OS in LUME-Lung 1 revealed a pronounced 3 month benefit in median OS with nintedanib plus docetaxel versus docetaxel alone in patients with adenocarcinoma and TSFLT $<9$ months (medians: 10.9 vs 7.9 months; HR: 0.75; 95\% CI: 0.60-0.92; $\mathrm{p}=0.0073)[23,33]$. Exploratory analyses of the LUME-Lung 1 adenocarcinoma population also indicated a more marked OS benefit of nintedanib in other aggressive disease subgroups, including TSFLT $<5$ or $<6$ months, TEFLT $<3$ or $\leq 6$ months, and PD-FLT [30,33]. Together, these results strongly suggest that adenocarcinoma patients with the poorest outcomes during first-line therapy derive the greatest benefit from second-line treatment with nintedanib in combination with docetaxel.

\section{Ramucirumab}

Ramucirumab is an anti-angiogenic monoclonal antibody against VEGFR2 that is approved for use in combination with docetaxel for treatment of advanced NSCLC that has progressed following platinum-containing chemotherapy [72]. In the primary end point analysis of the randomized Phase III REVEL study of 1253 patients with stage IV 
NSCLC, ramucirumab plus docetaxel significantly increased OS compared with placebo plus docetaxel medians: 10.5 vs 9.1 months; HR: 0.86 ; $95 \%$ CI: $0.75-0.98 ; p=0.023$ ) [24]. Prespecified subgroup analyses showed a significant OS treatment effect for ramucirumab in patients with TSFLT $<9$ months (HR: 0.75; 95\% CI: 0.64-0.88), which appeared greater than that in patients with TSFLT $\geq 9$ months (HR: 0.95; 95\% CI: 0.75-1.20) [24,25]. In contrast to LUME-Lung 1, PD-FLT did not show clear evidence of a predictive effect for OS, with a similar HR in this group and in patients with at least SD in first line [24,25].

\section{Bevacizumab}

The anti-VEGF monoclonal antibody bevacizumab is approved for use with first-line chemotherapy for nonsquamous advanced NSCLC [73]. Although not currently indicated in subsequent lines, bevacizumab has been investigated in combination with weekly paclitaxel for second- or third-line treatment of nonsquamous NSCLC in the Phase III IFCT-1103 ULTIMATE trial $(n=166)[26,73]$. Analysis of the primary end point demonstrated a significant increase in PFS with bevacizumab plus weekly paclitaxel compared with docetaxel alone (HR: 0.62; 95\% CI: 0.44-0.87; $\mathrm{p}=0.006$ ), although there was no significant difference in OS. Subgroup analyses suggested a more pronounced PFS treatment effect of bevacizumab plus weekly paclitaxel versus docetaxel in patients with TSFLT $<9$ months (HR: 0.46; 95\% CI: 0.26-0.84) than in patients with TSFLT $\geq 9$ months (HR: 0.70; 95\% CI: 0.45-1.09).

\section{Nivolumab}

The PD-1 inhibitor nivolumab is approved for single-agent use for advanced NSCLC previously treated with chemotherapy, following positive results from two separate randomized Phase III trials in PD-L1-unselected, nonsquamous and squamous NSCLC (CheckMate 057 and 017, respectively) [54,5574]. In the primary analysis of CheckMate 057 ( $\mathrm{n}=582)$, median OS was 12.2 months in the nivolumab arm and 9.4 months in the docetaxel arm (HR: 0.73 ; 95\% CI: 0.59-0.89; $\mathrm{p}=0.002)$ [54]. The primary analysis of CheckMate $017(\mathrm{n}=352)$ similarly showed a significant OS benefit favoring the nivolumab arm (median OS: 9.2 vs 6.0 months; HR: 0.59; 95\% CI: 0.44-0.79; $\mathrm{p}<0.001$ ) [55]. In nonsquamous NSCLC, PD-L1 level predicted efficacy of nivolumab versus docetaxel in CheckMate 057, and no significant difference in OS was evident among patients with low or absent PD-L1 expression (TPS $<1 \%$ ) [54,75].

In contrast to results for anti-angiogenic therapies, prespecified subgroup analyses of CheckMate 057 showed an apparent trend for greater OS benefit in patients with longer TEFLT (TEFLT > 6 months; HR: 0.46; 95\% CI: $0.27-0.79)$ than in patients with aggressive disease (TEFLT $<3$ months [HR: 0.85 ; 95\% CI: $0.67-1.08$ ] or 3-6 months [HR: 0.69; 95\% CI: 0.44-1.08]) [54]. A consistent trend was potentially also seen for TSFLT $\geq 12$ versus $<12$ months. A striking observation from this study was the numerically higher mortality rate during the first 3 months of follow-up in the nivolumab arm than in the docetaxel arm (20 vs 15\%, respectively), which was mostly due to PD-related deaths [27]. Post hoc exploratory multivariate analyses identified aggressive disease features (TSFLT $<3$ months and PD-FLT), along with Eastern Cooperative Oncology Group PS of 1, as risk factors for early mortality with nivolumab, when combined with low or absent PD-L1 expression [27].

Retrospective studies have provided some corroboration for these findings in real-world populations with nonsquamous and squamous NSCLC, who received nivolumab in second or subsequent lines, irrespective of PD-L1 expression [76-78]. In a Japanese study of 201 patients with nonsquamous or squamous NSCLC (any PD-L1 expression level) receiving nivolumab in second or later lines, 38 patients (19\%) died within the first 3 months of treatment [76]. Poor response to prior therapy was identified as an independent predictor of early death, along with Eastern Cooperative Oncology Group PS $\geq 2$ and high C-reactive protein-to-albumin ratio. Similarly, in a Spanish retrospective study of nivolumab in second or subsequent lines of therapy in 175 patients with advanced NSCLC, time since start of prior therapy of $<6$ months was significantly associated with poorer OS in multivariate analyses $(p=0.010)$ [77]. This variable was also associated with shorter PFS and lower ORR. In a French retrospective cohort of 115 patients, the disease control rate with nivolumab was significantly lower in those with PD-FLT than in those who achieved at least SD during first-line therapy (60 vs 20\%; $\mathrm{p}=0.002)$ [78]. PD-FLT was also significantly associated with shorter PFS and OS with nivolumab in multivariate analyses $(\mathrm{p}=0.002$ and $\mathrm{p}=0.03$, respectively). Finally, Japanese retrospective data showed that the disease control rate with nivolumab was significantly lower in patients with PD-FLT compared with those who previously achieved disease control with chemotherapy (35 vs $61 \% ; \mathrm{p}<0.001)[79]$. 


\section{Pembrolizumab}

In addition to its first-line indications, the PD- 1 inhibitor pembrolizumab is approved for use as monotherapy in patients with previously treated advanced NSCLC and PD-L1 TPS $\geq 1 \%$. In the Phase II/III KEYNOTE-010 trial $(\mathrm{n}=1034)$, the approved pembrolizumab dose $(2 \mathrm{mg} / \mathrm{kg})$ was associated with significant increases in the co-primary end point of OS, both in the overall population and in patients with PD-L1 TPS $\geq 50 \%$, when compared with docetaxel alone [80]. Median OS in the overall population was 10.4 months with pembrolizumab versus 8.5 months with docetaxel (HR: $0.71 ; 95 \%$ CI: $0.58-0.88 ; \mathrm{p}=0.0008$ ). The second co-primary end point of PFS showed a significant difference for pembrolizumab $2 \mathrm{mg} / \mathrm{kg}$ versus docetaxel in patients with PD-L1 TPS $\geq 50 \%$, but not in the overall population. Unlike CheckMate 057 , no increase in early death was evident in the immunotherapy arms. To our knowledge, no data have been presented regarding TSFLT or aggressive disease subgroups in this trial.

\section{Atezolizumab}

The most recently approved immunotherapy for NSCLC is the PD-L1 inhibitor atezolizumab, which is indicated for single-agent use in patients with prior platinum chemotherapy [81]. In the co-primary end point analyses of the first 850 patients randomized into the Phase III OAK trial, atezolizumab provided a significant increase in OS versus docetaxel alone in both the overall population (median OS: 13.8 vs 9.6 months; HR: 0.73; 95\% CI: 0.62-0.87; $\mathrm{p}=0.0003$ ) and the population with PD-L1 expression on $\geq 1 \%$ of tumor cells or $\geq 1 \%$ of the tumor area infiltrated by PD-L1-positive immune cells (15.7 vs 10.3 months; HR: 0.74; 95\% CI: 0.58-0.93; p = 0.0102) [82]. Although no association of atezolizumab with early death was evident, PFS was numerically shorter with atezolizumab than with docetaxel during the first months of follow-up, an effect that appeared to be driven by patients with lower PD-L1 expression. The potential relationship with features of prior aggressive disease behavior has not been reported to our knowledge.

\section{EGFR inhibitors}

Phase III trials of second-/third-line treatment with EGFR inhibitors have been conducted in aggressive disease populations but lack contemporary relevance due to their EGFR-unselected populations [37,49]. The ISEL study showed no significant difference in OS with gefitinib versus placebo in patients with progression or recurrence within 90 days of prior chemotherapy completion or intolerance to prior chemotherapy [37]. In patients with progression on or after four cycles of induction chemotherapy in the TITAN study, there was no significant difference in OS with erlotinib compared with docetaxel or pemetrexed [49]. Finally, OS with gefitinib versus docetaxel did not appear to differ according to TSFLT or PD-FLT subgroups in EGFR-unselected patients in the Phase III INTEREST trial [60].

\section{Pathophysiology of aggressive NSCLC}

The mechanistic basis of platinum resistance is complex and multifactorial [83]. Predominantly based on in vitro studies, several resistance mechanisms have been described, including poor drug uptake due to low copper transporter-1 expression, drug detoxification resulting from high glutathione S-transferase M1 activity and resistance to apoptosis via overexpression of prosurvival factors (e.g., B-cell lymphoma protein-2 or serpin B3) [7,84,85]. Some of the most extensively studied molecular determinants of platinum resistance involve components of the DNA repair pathways that can reverse the intra- and interstrand crosslinks introduced by platinum compounds $[7,84,86]$. Expression and/or genetic polymorphisms in ERCC1, BRCA1 and RRM1 have been correlated with outcomes of platinumbased chemotherapy, but none of these biomarkers have so far been prospectively validated for predictive use [86]. Chemoresistance has also been associated with certain rare NSCLC histological subtypes, including sarcomatoid carcinomas and invasive mucinous adenocarcinomas, although this may be partly anecdotal $[87,88]$.

Rapid progression of NSCLC is likely to be reflected in a high rate of cellular proliferation within the tumor. This is supported by a gene-set enrichment analysis of robust multi-array normalized gene expression data from a large database generated by the Director's Challenge Consortium for the Molecular Classification of Adenocarcinoma [89]. In that dataset, proliferation-related genes were more frequently expressed in patients with shorter OS compared with those who survived for longer after diagnosis. Consistent with this, the proportion of proliferating tumor cells, assessed according to expression of the proliferation marker Ki-67, has been associated with tumor size and nodal stage, as well as shorter OS in patients with adenocarcinoma NSCLC [90].

One of the consequences of rapid cancer growth is the development of a hostile tumor microenvironment characterized by hypoxia, low nutrient levels and acidification of the extracellular milieu [91-93]. Hypoxia, in 
particular, is thought to be a critical factor in the development of aggressive tumor characteristics [91,93]. Although intratumoral hypoxia may retard or limit cancer growth, it also triggers adaptive mechanisms that help to establish a vicious circle by promoting further tumor progression [94]. A well-characterized response to hypoxia is the upregulation of angiogenic factors, including VEGF, PDGF and FGF, which stimulate the formation of new vasculature. Rapidly progressing tumors may, therefore, be particularly dependent on this process for the delivery of adequate oxygen and nutrients, and so be more sensitive to anti-angiogenic therapies [23,30].

Furthermore, severe hypoxia can promote genomic instability while enhancing selective pressure, which may in turn foster the emergence of aggressive tumor characteristics, including chemoresistance and increased propensity to metastasize [94]. A number of microRNAs have been implicated in hypoxia-induced platinum resistance in NSCLC [83]. At the same time, hypoxia has been shown to modulate the immunological character of the microenvironment such that antitumor immunity may be downregulated [93]. VEGF, in addition to its pro-angiogenic properties, has several immunosuppressive actions, including inhibiting the maturation of granulocyte-macrophage and dendritic cell precursors and inhibiting the recruitment of M2 macrophages [93]. Thus, hypoxia and its downstream effects could be a key mechanism linking platinum resistance, sensitivity to anti-angiogenics and poor response to immunotherapies.

\section{Clinical implications \& definition of aggressive NSCLC Treatment selection}

At present, the most clinically relevant findings regarding aggressive NSCLC relate to its predictive potential for second-line targeted therapies (Table 3). TSFLT $<9$ months has been prospectively validated to identify patients who derive more pronounced benefit of nintedanib with second-line docetaxel for adenocarcinoma NSCLC, and the treatment effect appeared to increase still further with shorter TSFLT durations [23,30,33]. Exploratory analyses indicate this positive predictive relevance may similarly apply for other anti-angiogenic therapies, namely ramucirumab (irrespective of tumor histology) and bevacizumab (in nonsquamous NSCLC) [24-26,30,33]. The consistent signal across three independent Phase III trials suggests that neoangiogenesis plays a more important role in more rapidly progressing or aggressive lung tumors, but further data are required to confirm this dependency.

Conversely, features of aggressive disease have been associated with poor outcome with second-line nivolumab in patients with nonsquamous tumors and low or absent tumor PD-L1 expression, and in less selected real-world populations [27,76-78]. Some caution is required when interpreting the opposing directions of this effect with nivolumab relative to the anti-angiogenic compounds nintedanib, ramucirumab and bevacizumab. Many of the analyses are exploratory and/or post hoc and the apparent disparity may be explained by differences in patient populations. Furthermore, relevant data are available from only a single immunotherapy trial (CheckMate 057) and the impact of aggressive disease on other immunotherapies, such as pembrolizumab, requires further evaluation. Nonetheless, it has been suggested that anti-angiogenic therapies might be favored over immunotherapies in the second-line treatment of nonsquamous NSCLC with low PD-L1 expression and fast progression or lack of disease control with first-line platinum-containing therapy [95]. Such an approach has some precedent given the importance of platinum sensitivity in clinical decision making for other tumor types, including SCLC and ovarian cancer [96]. Although this treatment paradigm emerged from the correlation between the duration of the platinum-free interval and sensitivity to platinum rechallenge, its predictive relevance extends beyond platinum agents to other drugs with different mechanisms of action [97].

\section{Defining aggressive NSCLC}

One challenge in the clinical implementation of such a management model is the lack of accepted definition of aggressive NSCLC. The wide variety of definitions utilized so far specify distinct but overlapping subgroups, underscoring a need to develop consensus criteria by which patients with aggressive disease can be optimally identified. Based on a thorough review of the available clinical data, we recommend defining aggressive nonsquamous NSCLC based on TSFLT $<6-9$ months. Although the 9 month cut-off represents the best studied definition to date (Table 3), clinical observations of the authors suggest that the 6-month cut-off may be most useful for identifying this subgroup in practice. Published evidence and clinical experience to date indicate this criterion would identify a substantial proportion of patients in current clinical practice, whether treated in the first-line with chemotherapy alone, immunotherapy alone or regimens combining these modalities. In addition to the predictive considerations discussed above, these patients may benefit from intensified monitoring (e.g., every 2 weeks) due to the aggressive 


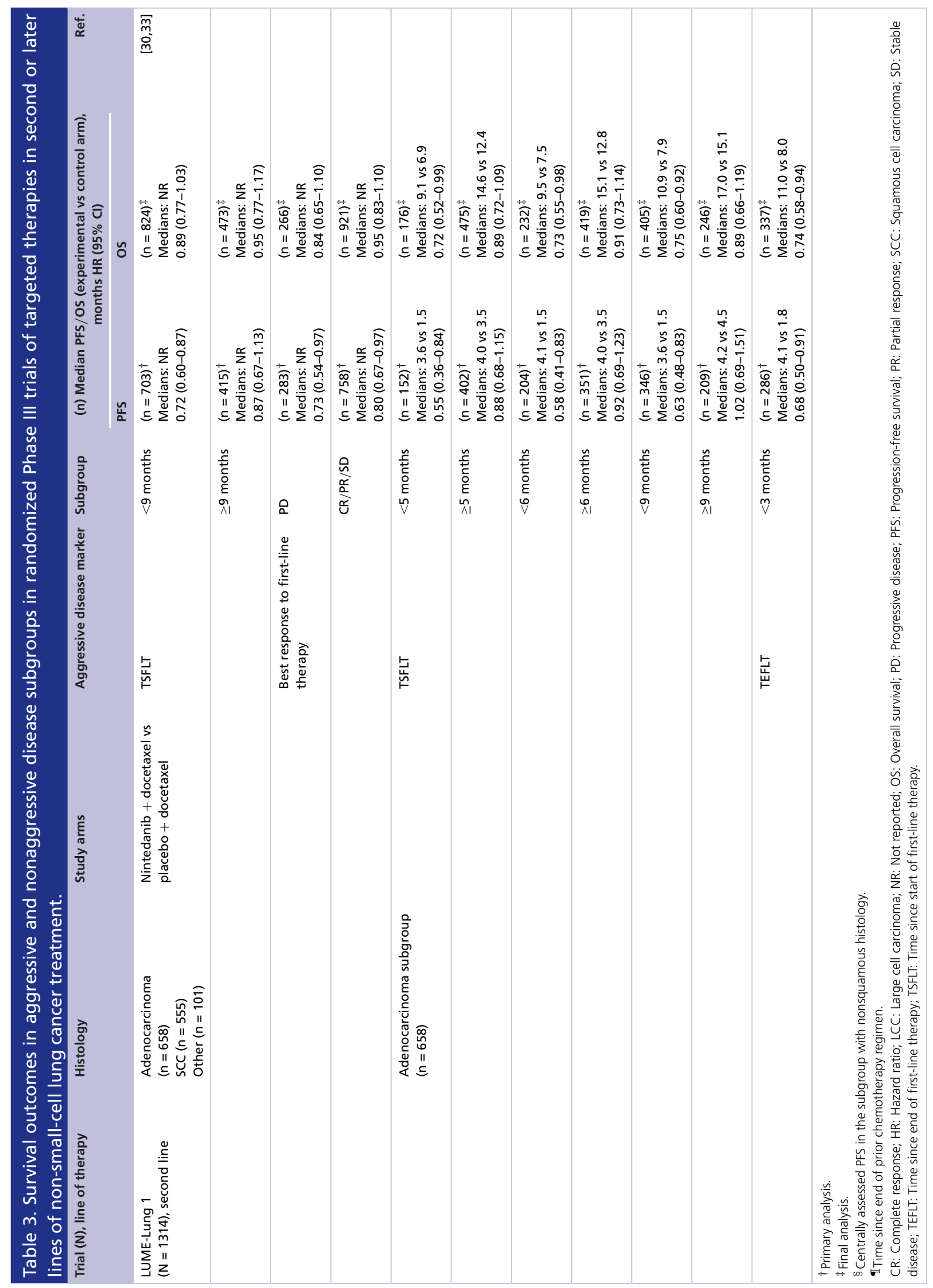




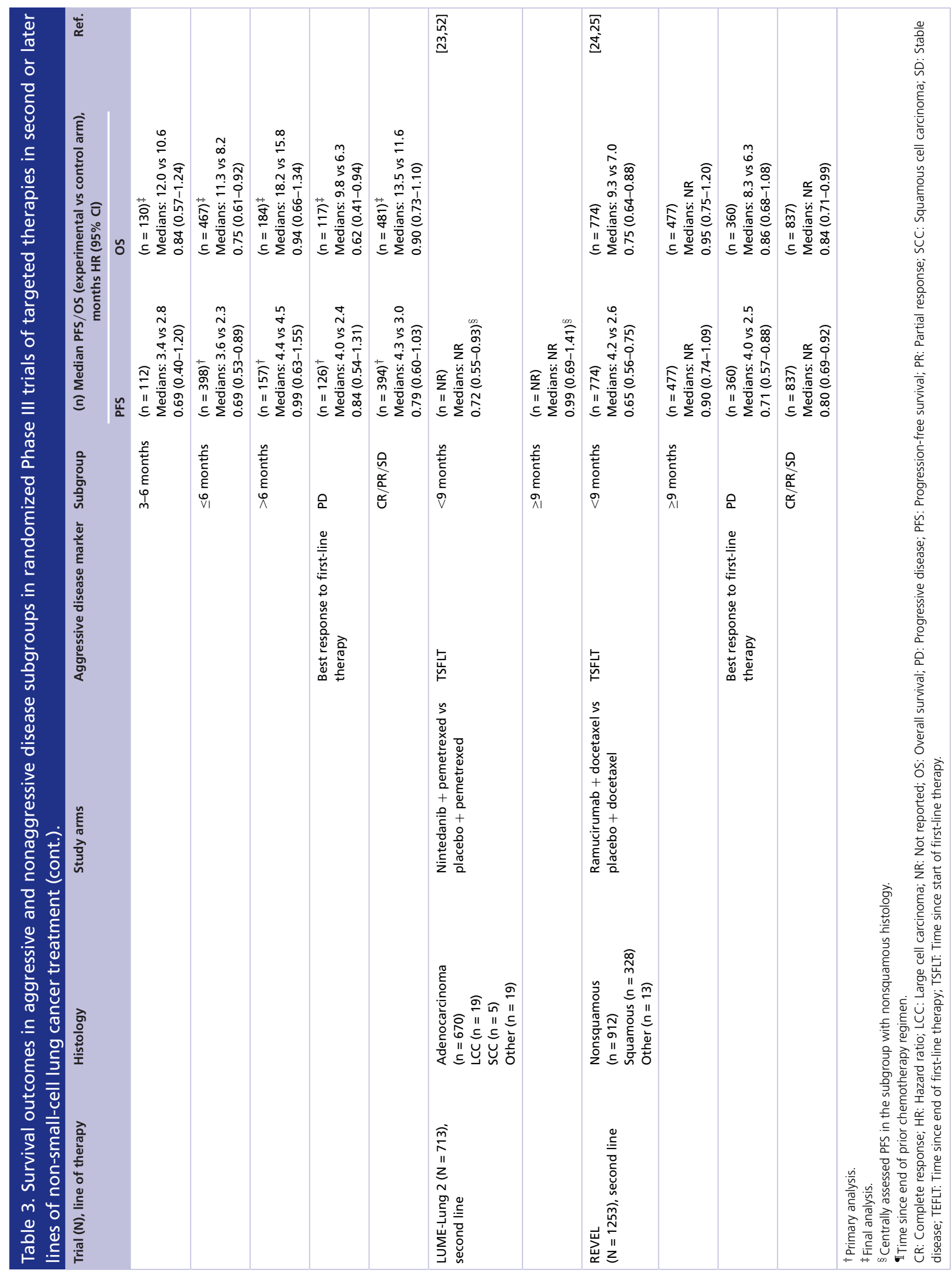




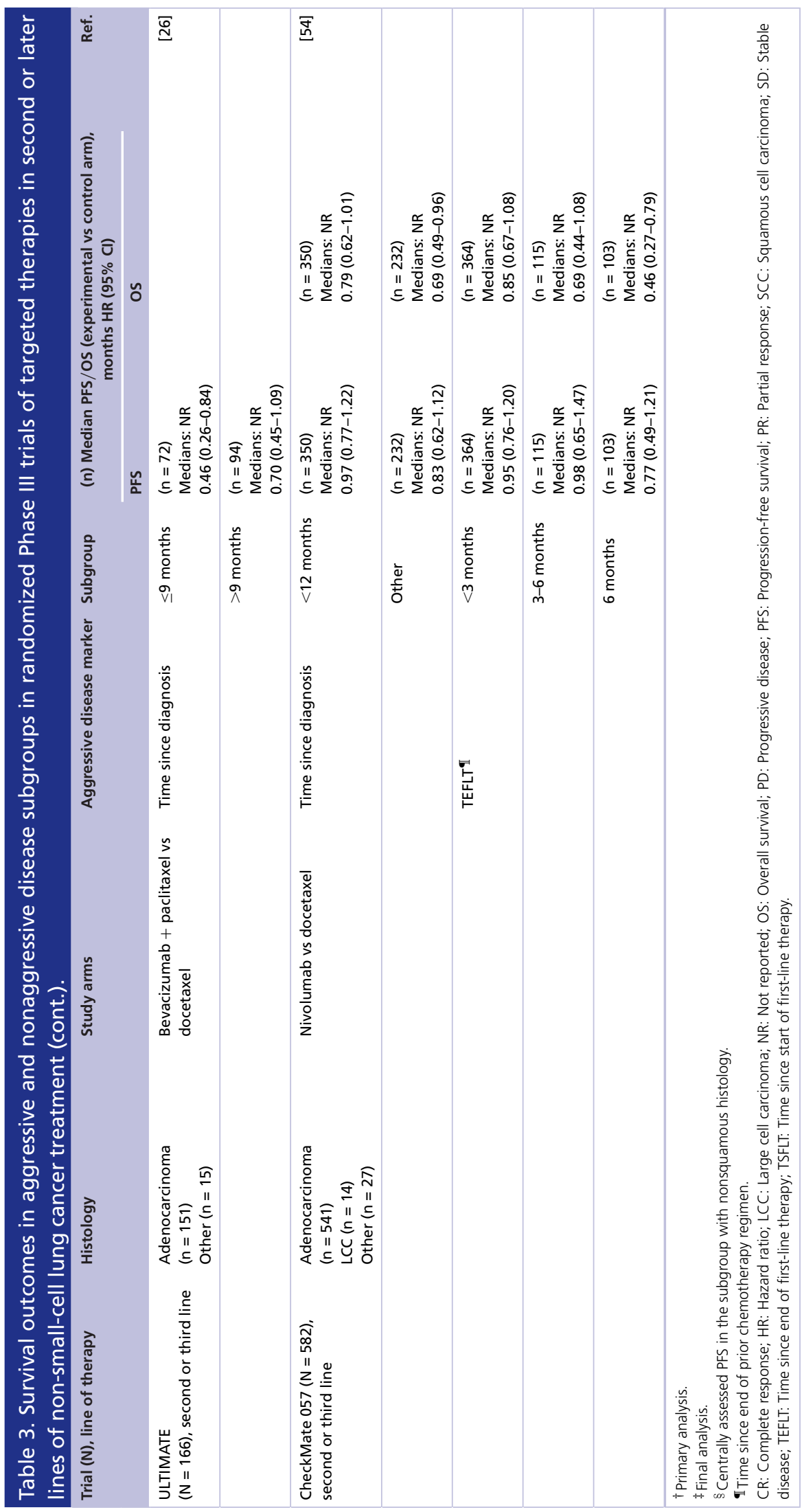


course of disease. It is likely that this patient population has a separate disease entity with a distinct genetic or immunological background, which requires further biologic and clinical investigation.

\section{Future directions}

Current definitions of aggressive NSCLC based on response to initial therapy do not enable up-front patient identification, nor do they distinguish between aggressive underlying disease and treatment refractoriness. These central limitations may be addressed through careful clinical, pathological and molecular characterization of patients with aggressive disease in order to improve our understanding of the biological determinants of treatment resistance, as well as the factors driving de novo aggressive disease behavior. One key goal will be the development of novel molecular or pathologically assessable morphological markers that can predict rapid disease progression and thereby enable appropriately tailored management from diagnosis.

Our clinical definition of aggressive disease can support this endeavor by harmonizing research based on more uniform populations of patients with rapidly progressing disease. Given the mounting evidence that aggressive disease has both prognostic and predictive relevance, we recommend that future studies should prespecify analyses of this aggressive disease subgroup. In this respect, results are keenly awaited from the ongoing noninterventional LUME-BioNIS study, which is investigating associations between a wide range of biomarkers and TSFLT $<9$ months in patients receiving nintedanib in line with the approved label [98]. Ultimately, it will be of interest to evaluate novel treatment strategies for this patient group, such as early anti-angiogenic therapy plus a taxane or combinations of anti-angiogenic agents with immunotherapies.

\section{Conclusion}

It is clear that a substantial subpopulation of patients with nononcogene-addicted advanced NSCLC have an aggressive course of disease, with resistance to first-line platinum doublets or immunotherapy with or without chemotherapy. These patients have been under-recognized to date and have a high unmet medical need for new treatment options that could transform their poor prognosis. Emerging data suggest that features of aggressive disease differentially impact on the efficacy of some targeted therapies in the second-line setting. Further investigations are required to improve our understanding of the pathophysiological mechanisms driving aggressive disease, which may represent a distinct disease entity. Our proposed definition of aggressive NSCLC can support research aiming to advance the care of this neglected patient subgroup.

\section{Future perspective}

Over the next 5-10 years, better understanding of the molecular biology of NSCLC will continue to drive the division of the disease into different subtypes, with a consequent increase in the complexity of management and therapeutic considerations. To fully exploit the new opportunities for personalized medicine, clinical trial designs will require adaptation to enable meaningful analyses of large numbers of potentially prognostic and predictive factors. In this respect, alongside novel molecular predictors, clinical variables such as TSFLT $<9$ months may be valuable. In addition to new molecular biomarkers, the development of novel pathologically assessable morphological markers may help address the urgent need to enable up-front identification of chemoresistant and/or aggressive disease. We anticipate that improved definition and recognition of this patient subgroup will ultimately expand the available range of effective strategies, which may include novel combinations and/or rational treatment sequencing.

\section{Executive summary}

- Platinum-based chemotherapy was for many years the mainstay of first-line treatment for nononcogene-addicted non-small-cell lung cancer (NSCLC); however, the treatment paradigm is currently evolving with the incorporation of immunotherapies into this setting.

- Platinum doublets provide an overall response rate of $20-30 \%$ and median time to progression of 3.5-5.5 months, but almost all patients will eventually develop progressive disease.

- Second-line options can include anti-angiogenic agents (nintedanib or ramucirumab) plus docetaxel, single-agent immunotherapies (pembrolizumab, nivolumab or atezolizumab) or cytotoxic monotherapy (docetaxel and pemetrexed), depending on first-line therapy, tumor histology and PD-L1 expression.

- Recently, clinical correlates of aggressive disease behavior during first-line treatment have been shown to predict greater benefit of second-line nintedanib in combination with docetaxel in patients with adenocarcinoma NSCLC. The predictive relevance may also extend to other agents. 
- Despite its emerging predictive significance, there is no standard definition of 'aggressive NSCLC' and these patients represent an under-recognized and neglected population who have poor outcomes with current therapy. Concept of aggressive NSCLC

- 'Aggressive disease' refers to tumors that develop, progress or metastasize more quickly, manifesting as poor clinical outcomes, including lack of disease control and shorter time to progression.

- Analyses to date have defined aggressive disease subgroups based on shorter time since start of first-line therapy (TSFLT, with $<9$ months as the most commonly used cut-point), time since end of first-line therapy or progressive disease as best response to first-line therapy (PD-FLT).

- The term 'hyperprogressive disease' refers to the paradoxical acceleration of tumor growth during treatment with immunotherapies, relative to the pretreatment period.

Prevalence of aggressive NSCLC

- A body of evidence indicates that a significant proportion of patients with NSCLC derive no or only limited benefit from first-line chemotherapy.

- Control arms of recent first-line immunotherapy studies demonstrate a 10-27\% rate of PD-FLT with platinum-based doublets in patient populations selected according to tumor histology and molecular characteristics.

- In maintenance therapy trials, $14-31 \%$ of patients had progressive disease after 3-4 months of platinum-based induction chemotherapy.

- Baseline data from recent second-line trials indicate thatup to $62 \%$ of patients have TSFLT $<9$ months and up to $39 \%$ have PD-FLT in populations considered suitable for further therapy.

Prognosis of aggressive NSCLC

- Both randomized controlled trials and observational studies indicate that aggressive disease during first-line therapy has a negative prognostic impact on outcomes in subsequent treatment lines.

Demographic \& clinical characteristics of patients with aggressive NSCLC

- Few studies have reported correlations between aggressive disease and demographic or clinical variables.

- Lack of disease control in first-line treatment has been associated with the presence of adrenal metastases, poor performance status and weight loss at diagnosis in an observational study.

Predictive relevance of aggressive NSCLC for efficacy of targeted therapies in second \& subsequent lines

- The predictive relevance of aggressive disease was first highlighted in a systematic evaluation of LUME-Lung 2, which identified TSLFT $<9$ months as a potential positive predictive marker for nintedanib in combination with second-line chemotherapy.

- TSFLT $<9$ months was subsequently confirmed to predict the treatment effect of second-line nintedanib plus docetaxel versus placebo plus docetaxel in a prespecified analysis of overall survival (OS) in patients with adenocarcinoma histology in LUME-Lung 1.

- The addition of nintedanib to docetaxel provided a pronounced 3 month benefit in median OS (median of 10.9 vs 7.9 months with docetaxel alone; hazard ratio: $0.75 ; 95 \% \mathrm{Cl}: 0.60-0.92 ; \mathrm{p}=0.0073$ ). Exploratory analyses also showed a more marked OS benefit of nintedanibin other aggressive disease subgroups including patients with PD-FLT.

- These observations have since been extended in subgroup analyses of other Phase III trials, which suggest that TSFLT $<9$ months also predicts greater efficacy of ramucirumab in combination with docetaxel (REVEL; any histology) and bevacizumab plus weekly paclitaxel (ULTIMATE; nonsquamous histology).

- Conversely, aggressive disease features (TSFLT $<3$ months and PD-FLT), along with poorer performance status, were identified as risk factors for early mortality with nivolumab, when combined with low or absent PD-L1 expression in an exploratory analysis in nonsquamous NSCLC (CheckMate 057).

- To our knowledge, no aggressive disease subgroup data have been reported for second-line trials of pembrolizumab or atezolizumab.

Pathophysiology of aggressive NSCLC

- Proposed chemoresistance mechanisms include poor drug uptake due to low copper transporter-1 expression, drug detoxification resulting from high glutathione S-transferase M1 activity, and resistance to apoptosis via overexpression of prosurvival factors.

- Due to a rapid rate of cellular proliferation, aggressive tumors might be particularly dependent on neoangiogenesis for the delivery of adequate oxygen and nutrients, which may in turn sensitize them to anti-angiogenic therapies.

Clinical implications \& definition of aggressive NSCLC

- Given the opposing directions of the predictive effect of aggressive disease for anti-angiogenic therapies (plus a taxane) compared with nivolumab, it has suggested that anti-angiogenic therapy may be preferred over immunotherapies in the second-line treatment of nonsquamous NSCLC with low PD-L1 expression.

- We recommend defining aggressive nonsquamous NSCLC based on TSFLT $<6-9$ months, which is expected to identify a substantial proportion of patients in current practice, irrespective of initial treatment type. 
- Further investigation is required to elucidate the pathophysiological basis of aggressive disease behavior and identify novel molecular and/or pathologically assessable morphological markers to enable up-front patient recognition.

\section{Conclusion}

- A substantial proportion of advanced NSCLCs shows aggressive disease behavior during first-line therapy, which is associated with a poor prognosis and may differentially impact on the efficacy of second-line targeted therapies.

Acknowledgements

S Popat acknowledges NHS funding to the Royal Marsden Hospital/Institute of Cancer Research NIHR Biomedical Research Centre.

Financial \& competing interests disclosure

$M$ Reck has received fees for honoraria, consulting/advisory roles and speaker's bureau from Boehringer Ingelheim, F Hoffmann-La Roche, Lilly, AstraZeneca, BMS, MSD, Merck, Novartis, Pfizer and Celgene. KM Kerr has received advisor's fees and/or payment for speaking from AbbVie, Archer Diagnostics, AstraZeneca, Bayer, BMS, Boehringer Ingelheim, Eli Lilly, Merck Serono, MSD, Novartis, Pfizer, Roche, Roche Diagnostics (Ventana). C Grohé has received personal fees and nonfinancial support from Boehringer Ingelheim Germany, personal fees from Lilly Germany, Roche Germany, Pfizer Germany, BMS Germany, MSD Germany, AstraZeneca, Novartis Germany, Ariad and Clovis. C Manegold has no conflicts of interest to disclose. N Pavlakis has received fees for advisory boards from BMS, Merck-KgA, Boehringer Ingelheim, AstraZeneca, Roche, Bayer, Novartis, Merck, Pfizer and Takeda, research funding from Bayer and Pfizer and travel funding from Boehringer Ingelheim, BMS and Roche. $L$ Paz-Ares has received personal fees for advisory boards from Roche, MSD, Boehringer Ingelheim, BMS, AstraZeneca, Lilly, Clovis Oncology, Pfizer, Novartis and Amgen. RM Huber has received personal fees and nonfinancial support from Boehringer Ingelheim Germany, personal fees from Lilly Germany, Roche Germany, Pfizer Germany, BMS Germany, MSD Germany, AstraZeneca, Novartis Germany, Ariad and Clovis. S Popat has received nonfinancial editorial support from Millennium Pharmaceuticals outside of the submitted work and received institutional research funding from Boehringer Ingelheim, Epizyme, BMS, Clovis Oncology, Roche, Lilly, Takeda and Pfizer, fees and nonfinancial support for travel, accommodations and expenses from Boehringer Ingelheim, fees, and nonfinancial support for consulting roles, travel, accommodations and expenses from BMS, fees for honoraria and consulting roles from Roche and AstraZeneca, fees from Takeda and Chugai Pharma, fees for consulting roles from Novartis, Pfizer, Guardant Health and AbbVie, and nonfinancial support for consulting roles, travel, accommodations and expenses from MSD. N Thatcher has received personal fees from Amgen, Lilly, AstraZeneca, Roche and Boehringer Ingelheim. K Park reports an advisory role from Boehringer Ingelheim. F Hilberg and J Barrueco are employees of Boehringer Ingelheim. R Kaiser is an employee of Boehringer Ingelheim and has a patent EP 2994125 issued. The authors have no other relevant affiliations or financial involvement with any organization or entity with a financial interest in or financial conflict with the subject matter or materials discussed in the manuscript apart from those disclosed.

Medical writing assistance, supported financially by Boehringer Ingelheim, was provided by M Dyson on behalf of Syneos Health (London, UK), during the preparation of this manuscript.

\section{Open access}

This work is licensed under theAttribution-NonCommercial-NoDerivatives 4.0 Unported License. To view a copy of this license, visit http://creativecommons.org/licenses/by-nc-nd/4.0/

\section{References}

Papers of special note have been highlighted as: $\bullet$ of interest; $\bullet \bullet$ of considerable interest

1. Ramalingam SS, Owonikoko TK, Khuri FR. Lung cancer: new biological insights and recent therapeutic advances. CA Cancer J. Clin. 61(2), 91-112 (2011).

2. Ahmadzada T, Kao S, Reid G, Boyer M, Mahar A, Cooper WA. An update on predictive biomarkers for treatment selection in non-small cell lung cancer. J. Clin. Med. 7(6), pii:E153 (2018).

3. Barlesi F, Mazieres J, Merlio J-P et al. Routine molecular profiling of patients with advanced non-small-cell lung cancer: results of a 1 year nationwide programme of the French Cooperative Thoracic Intergroup (IFCT). Lancet 387(10026), 1415-1426 (2016).

4. Planchard D, Popat S, Kerr K et al. Metastatic non-small cell lung cancer: ESMO Clinical Practice Guidelines for diagnosis, treatment and follow-up. Ann. Oncol. 29(Suppl. 4), iv192-iv237 (2018).

5. Leighl NB. Treatment paradigms for patients with metastatic non-small-cell lung cancer: first-, second-, and third-line. Curr. Oncol. 19(Suppl. 1), S52-S58 (2012).

6. Aggarwal C, Somaiah N, Simon GR. Biomarkers with predictive and prognostic function in non-small cell lung cancer: ready for prime time? J. Natl Compr. Canc. Netw. 8(7), 822-832 (2010). 
7. Olaussen KA, Postel-Vinay S. Predictors of chemotherapy efficacy in non-small-cell lung cancer: a challenging landscape. Ann. Oncol. 27(11), 2004-2016 (2016).

8. Soria J-C, Mauguen A, Reck M et al. Systematic review and meta-analysis of randomised, Phase II/III trials adding bevacizumab to platinum-based chemotherapy as first-line treatment in patients with advanced non-small-cell lung cancer. Ann. Oncol. 24(1), 20-30 (2013).

9. Perez-Moreno P, Brambilla E, Thomas R, Soria J-C. Squamous cell carcinoma of the lung: molecular subtypes and therapeutic opportunities. Clin. Cancer Res. 18(9), 2443-2451 (2012).

10. Reck M, Rodríguez-Abreu D, Robinson AG et al. Pembrolizumab versus chemotherapy for PD-L1-positive non-small-cell lung cancer. N. Engl. J. Med. 375(19), 1823-1833 (2016).

11. Gandhi L, Rodríguez-Abreu D, Gadgeel S et al. Pembrolizumab plus chemotherapy in metastatic non-small-cell lung cancer. N. Engl. J. Med. 378(22), 2078-2092 (2018).

12. Socinski MA, Jotte RM, Cappuzzo F et al. Atezolizumab for first-line treatment of metastatic nonsquamous NSCLC. N. Engl. J. Med. 378(24), 2288-2301 (2018).

13. Hellmann MD, Ciuleanu T-E, Pluzanski A et al. Nivolumab plus ipilimumab in lung cancer with a high tumor mutational burden. $N$. Engl. J. Med. 378(22), 2093-2104 (2018).

14. European Medicines Agency. Pembrolizumab Summary of Product Characteristics. (2018). www.ema.europa.eu/documents/product-information/keytruda-epar-product-information_en.pdf

15. Carbone DP, Reck M, Paz-Ares L et al. First-line nivolumab in stage IV or recurrent non-small-cell lung cancer. N. Engl. J. Med. 376(25), 2415-2426 (2017).

16. Paz-Ares L, Luft A, Vicente D et al. Pembrolizumab plus chemotherapy for squamous non-small-cell lung cancer. N. Engl. J. Med. 379(21), 2040-2051 (2018).

17. Jotte RM, Cappuzzo F, Vynnychenko I et al. IMpower131: primary PFS and safety analysis of a randomized Phase III study of atezolizumab + carboplatin + paclitaxel or nab-paclitaxel vs carboplatin + nab-paclitaxel as 1L therapy in advanced squamous NSCLC. J. Clin. Oncol. 36(18 Suppl.), Abstract LBA9000 (2018).

18. Borghaei H, Hellmann MD, Paz-Ares LG et al. Nivolumab (Nivo) + platinum-doublet chemotherapy (Chemo) vs chemo as first-line (1L) treatment (Tx) for advanced non-small cell lung cancer (NSCLC) with $<1 \%$ tumor PD-L1 expression: results from CheckMate 227. J. Clin. Oncol. 36(15 Suppl.), Abstract 9001 (2018).

19. Manegold C, Adjei A, Bussolino F et al. Novel active agents in patients with advanced NSCLC without driver mutations who have progressed after first-line chemotherapy. ESMO Open 1(6), e000118 (2016).

20. Stinchcombe TE, Socinski MA. Considerations for second-line therapy of non-small cell lung cancer. Oncologist 13(Suppl. 1), 28-36 (2008).

21. Davies J, Patel M, Gridelli C, de Marinis F, Waterkamp D, McCusker ME. Real-world treatment patterns for patients receiving second-line and third-line treatment for advanced non-small cell lung cancer: a systematic review of recently published studies. PLoS ONE 12(4), e0175679 (2017).

22. Kulkarni S, Vella ET, Coakley N et al. The use of systemic treatment in the maintenance of patients with non-small cell lung cancer: a systematic review. J. Thorac. Oncol. 11(7), 989-1002 (2016).

23. Gaschler-Markefski B, Sikken P, Heymach JV et al. Time since start of first-line therapy as a predictive clinical marker for nintedanib in patients with previously treated non-small cell lung cancer. ESMO Open 2(1), e000102 (2017).

-. Systematic analyses of the Phase III LUME-Lung 1 and LUME Lung-2 trials, showing initial identification and validation of time since start of first-line therapy (TSFLT) $<9$ months as a predictive clinical marker for nintedanib in combination with second-line chemotherapy.

24. Garon EB, Ciuleanu T-E, Arrieta O et al. Ramucirumab plus docetaxel versus placebo plus docetaxel for second-line treatment of stage IV non-small-cell lung cancer after disease progression on platinum-based therapy (REVEL): a multicentre, double-blind, randomized Phase III trial. Lancet 384(9944), 665-673 (2014).

25. Reck M, Paz-Ares L, Bidoli P et al. Outcomes in patients with aggressive or refractory disease from REVEL: a randomized Phase III study of docetaxel with ramucirumab or placebo for second-line treatment of stage IV non-small-cell lung cancer. Lung Cancer 112, 181-187 (2017).

-• Exploratory analysis of the Phase III REVEL trial, showing greater treatment effect of ramucirumab plus docetaxel versus placebo plus docetaxel in patients with TSFLT $<9$ months in second-line treatment of non-small-cell lung cancer (NSCLC).

26. Cortot AB, Audigier-Valette CA, Molinier O et al. Weekly paclitaxel plus bevacizumab versus docetaxel as second or third-line treatment in advanced non-squamous non-small cell lung cancer (NSCLC): results from the Phase III study IFCT-1103 ULTIMATE. J. Clin. Oncol. 34(15 Suppl.), Abstract 9005 (2016).

27. Peters S, Cappuzzo F, Horn L et al. OA03.05 analysis of early survival in patients with advanced non-squamous NSCLC treated with nivolumab vs docetaxel in CheckMate 057. Presented at: IASLC 17th World Conference on Lung Cancer (WCLC). Vienna, Austria, 4-7 December (2016). 
-• Exploratory analysis of the Phase III CheckMate 057 trial associating features of aggressive disease with risk of early mortality with nivolumab versus docetaxel when combined with low PD-L1 expression in second-line treatment of nonsquamous NSCLC.

28. National Cancer Institute. Dictionary of cancer terms. www.cancer.gov/publications/dictionaries/cancer-terms

29. Arpino G, Milano M, De Placido S. Features of aggressive breast cancer. Breast 24(5), 594-600 (2015).

30. Gottfried M, Bennouna J, Bondarenko I et al. Efficacy and safety of nintedanib plus docetaxel in patients with advanced lung adenocarcinoma: complementary and exploratory analyses of the Phase III LUME-Lung 1 study. Target. Oncol. 12(4), 475-485 (2017).

- Exploratory analyses of LUME-Lung 1 suggesting more pronounced efficacy of nintedanib plus docetaxel versus placebo plus docetaxel in aggressive disease subgroups other than TSFLT $<9$ months.

31. Davis A, Tinker AV, Friedlander M. 'Platinum resistant' ovarian cancer: what is it, who to treat and how to measure benefit? Gynecol. Oncol. 133(3), 624-631 (2014).

32. Ardizzoni A, Tiseo M, Boni L. Validation of standard definition of sensitive versus refractory relapsed small cell lung cancer: a pooled analysis of topotecan second-line trials. Eur. J. Cancer 50(13), 2211-2218 (2014).

33. Reck M, Kaiser R, Mellemgaard A et al. Docetaxel plus nintedanib versus docetaxel plus placebo in patients with previously treated non-small-cell lung cancer (LUME-Lung 1): a Phase III, double-blind, randomized controlled trial. Lancet Oncol. 15(2), 143-155 (2014).

-. Primary analysis of the Phase III LUME-Lung 1 trial, including external validation of TSFLT $<9$ months as a predictor of pronounced overall survival benefit of nintedanib plus docetaxel versus placebo plus docetaxel in patients with adenocarcinoma NSCLC.

34. Scagliotti GV, Parikh P, von Pawel J et al. Phase III study comparing cisplatin plus gemcitabine with cisplatin plus pemetrexed in chemotherapy-naive patients with advanced-stage non-small-cell lung cancer. J. Clin. Oncol. 26(21), 3543-3551 (2008).

35. Moro-Sibilot D, Smit E, de Castro Carpeño J et al. Outcomes and resource use of non-small cell lung cancer (NSCLC) patients treated with first-line platinum-based chemotherapy across Europe: FRAME prospective observational study. Lung Cancer 88(2), 215-222 (2015).

36. Zhong W-Z, Zhou Q, Wu Y-L. The resistance mechanisms and treatment strategies for EGFR-mutant advanced non-small-cell lung cancer. Oncotarget 8(41), 71358-71370 (2017).

37. Thatcher N, Chang A, Parikh P et al. Gefitinib plus best supportive care in previously treated patients with refractory advanced non-small-cell lung cancer: results from a randomized, placebo-controlled, multicentre study (Iressa survival evaluation in lung cancer). Lancet 366(9496), 1527-1537 (2005).

38. Kurman JS, Murgu SD. Hyperprogressive disease in patients with non-small cell lung cancer on immunotherapy. J. Thorac. Dis. 10(2), 1124-1128 (2018).

39. Ferrara $\mathrm{R}$, Caramella C, Texier $\mathrm{M}$ et al. Hyperprogressive disease (HPD) is frequent in non-small cell lung cancer (NSCLC) patients (pts) treated with anti PD1/PD-L1 monoclonal antibodies (IO). Ann. Oncol. 28(Suppl. 5), 464 Abstract 1306PD (2017).

40. Schiller JH, Harrington D, Belani CP et al. Comparison of four chemotherapy regimens for advanced non-small-cell lung cancer. $N$. Engl. J. Med. 346(2), 92-98 (2002).

41. Scagliotti GV, De Marinis F, Rinaldi M et al. Phase III randomized trial comparing three platinum-based doublets in advanced non-small-cell lung cancer. J. Clin. Oncol. 20(21), 4285-4291 (2002).

42. Fossella F, Pereira JR, von Pawel J et al. Randomized, multinational, Phase III study of docetaxel plus platinum combinations versus vinorelbine plus cisplatin for advanced non-small-cell lung cancer: the TAX 326 study group. J. Clin. Oncol. 21(16), 3016-3024 (2003).

43. Kelly K, Crowley J, Bunn PA et al. Randomized Phase III trial of paclitaxel plus carboplatin versus vinorelbine plus cisplatin in the treatment of patients with advanced non-small-cell lung cancer: a Southwest Oncology Group trial. J. Clin. Oncol. 19(13), 3210-3218 (2001).

44. Thatcher N, Hirsch FR, Luft AV et al. Necitumumab plus gemcitabine and cisplatin versus gemcitabine and cisplatin alone as first-line therapy in patients with stage IV squamous non-small-cell lung cancer (SQUIRE): an open-label, randomised, controlled Phase III trial. Lancet Oncol. 16(7), 763-774 (2015).

45. Pérol M, Chouaid C, Pérol D et al. Randomized, Phase III study of gemcitabine or erlotinib maintenance therapy versus observation, with predefined second-line treatment, after cisplatin-gemcitabine induction chemotherapy in advanced non-small-cell lung cancer. $J$. Clin. Oncol. 30(28), 3516-3524 (2012).

46. Barlesi F, Scherpereel A, Rittmeyer A et al. Randomized Phase III trial of maintenance bevacizumab with or without pemetrexed after first-line induction with bevacizumab, cisplatin, and pemetrexed in advanced nonsquamous non-small-cell lung cancer: AVAPERL (MO22089). J. Clin. Oncol. 31(24), 3004-3011 (2013).

47. Fidias PM, Dakhil SR, Lyss AP et al. Phase III study of immediate compared with delayed docetaxel after front-line therapy with gemcitabine plus carboplatin in advanced non-small-cell lung cancer. J. Clin. Oncol. 27(4), 591-598 (2009).

48. Paz-Ares LG, de Marinis F, Dediu M et al. PARAMOUNT: final overall survival results of the Phase III study of maintenance pemetrexed versus placebo immediately after induction treatment with pemetrexed plus cisplatin for advanced nonsquamous non-small-cell lung cancer. J. Clin. Oncol. 31(23), 2895-2902 (2013). 
49. Ciuleanu T, Stelmakh L, Cicenas S et al. Efficacy and safety of erlotinib versus chemotherapy in second-line treatment of patients with advanced, non-small-cell lung cancer with poor prognosis (TITAN): a randomized multicentre, open-label, Phase III study. Lancet Oncol. 13(3), 300-308 (2012).

50. Belani CP, Barstis J, Perry MC et al. Multicenter, randomized trial for stage IIIB or IV non-small-cell lung cancer using weekly paclitaxel and carboplatin followed by maintenance weekly paclitaxel or observation. J. Clin. Oncol. 21(15), 2933-2939 (2003).

51. Brodowicz T, Krzakowski M, Zwitter M et al. Cisplatin and gemcitabine first-line chemotherapy followed by maintenance gemcitabine or best supportive care in advanced non-small cell lung cancer: a Phase III trial. Lung Cancer 52(2), 155-163 (2006).

52. Hanna NH, Kaiser R, Sullivan RN et al. Nintedanib plus pemetrexed versus placebo pluspemetrexed in patients with relapsed or refractory, advanced non-small cell lung cancer (LUME-Lung 2): a randomized, double-blind, Phase III trial. Lung Cancer 102, 65-73 (2016).

53. Shepherd FA, Rodrigues Pereira J, Ciuleanu T et al. Erlotinib in previously treated non-small-cell lung cancer. N. Engl. J. Med. 353(2), 123-132 (2005).

54. Borghaei H, Paz-Ares L, Horn L et al. Nivolumab versus docetaxel in advanced non squamous non-small-cell lung cancer. N. Engl. J. Med 373(17), 1627-1639 (2015).

55. Brahmer J, Reckamp KL, Baas P et al. Nivolumab versus docetaxel in advanced squamous-cell non-small-cell lung cancer. $N$. Engl. J. Med 373(2), 123-135 (2015).

56. Hanna N, Shepherd FA, Fossella FV et al. Randomized Phase III trial of pemetrexed versus docetaxel in patients with non-small-cell lung cancer previously treated with chemotherapy. J. Clin. Oncol. 22(9), 1589-1597 (2004).

57. Weiss GJ, Rosell R, Fossella F et al. The impact of induction chemotherapy on the outcome of second-line therapy with pemetrexed or docetaxel in patients with advanced non-small-cell lung cancer. Ann. Oncol. 18(3), 453-460 (2007).

- Data from the Phase III JMEI trial showing negative prognostic impact of aggressive disease during first-line therapy on outcomes in subsequent treatment lines.

58. Shepherd FA, Dancey J, Ramlau R et al. Prospective randomized trial of docetaxel versusbest supportive care in patients with non-small-cell lung cancer previously treated with platinum-based chemotherapy. J. Clin. Oncol. 18(10), 2095-2103 (2000).

59. Fossella FV, DeVore R, Kerr RN et al. Randomized Phase III trial of docetaxel versus vinorelbine or ifosfamide in patients with advanced non-small-cell lung cancer previously treated with platinum-containing chemotherapy regimens. The TAX 320 Non-Small Cell Lung Cancer Study Group. J. Clin. Oncol. 18(12), 2354-2362 (2000).

60. Kim ES, Hirsh V, Mok $\mathrm{T}$ et al. Gefitinib versus docetaxel in previously treated non-small-cell lung cancer (INTEREST): a randomised Phase III trial. Lancet. 372(9652), 1809-1818 (2008).

61. Herbst RS, Sun Y, Eberhardt WEE et al. Vandetanib plus docetaxel versus docetaxel as second-line treatment for patients with advanced non-small-cell lung cancer (ZODIAC): a double-blind, randomized, Phase 3 trial. Lancet Oncol. 11(7), 619-626 (2010).

62. Herbst RS, Ansari R, Bustin F et al. Efficacy of bevacizumab plus erlotinib versus erlotinib alone in advanced non-small-cell lung cancer after failure of standard first-line chemotherapy (BeTa): a double-blind, placebo-controlled, Phase 3 trial. Lancet. 377(9780), 1846-1854 (2011).

63. Garassino MC, Martelli O, Broggini M et al. Erlotinib versus docetaxel as second-line treatment of patients with advanced non-small-cell lung cancer and wild-type EGFR tumours (TAILOR): a randomised controlled trial. Lancet Oncol. 14(10), 981-988 (2013).

64. Ramlau R, Gervais R, Krzakowski M et al. Phase III study comparing oral topotecan to intravenous docetaxel in patients with pretreated advanced non-small-cell lung cancer. J. Clin. Oncol. 24(18), 2800-2807 (2006).

65. Bischoff HG, van den Borne B, Pimentel FL et al. Observation of the treatment and outcomes of patients receiving chemotherapy for advanced NSCLC in Europe (ACTION study). Curr. Med. Res. Opin. 26(6), 1461-1470 (2010).

66. Zietemann V, Duell T. Prevalence and effectiveness of first-, second-, and third-line systemic therapy in a cohort of unselected patients with advanced non-small cell lung cancer. Lung Cancer 73(1), 70-77 (2011).

67. Reinmuth N, Payer N, Muley T et al. Treatment and outcome of patients with metastatic NSCLC: a retrospective institution analysis of 493 patients. Respir. Res. 14, 139 (2013).

- Retrospective German data showing negative prognostic impact of TSFLT $<9$ months and progressive disease as best response to first-line therapy in a real-world patient population.

68. Florescu M, Hasan B, Seymour L, Ding K, Shepherd FA, National Cancer Institute of Canada Clinical Trials Group. A clinical prognostic index for patients treated with erlotinib in National Cancer Institute of Canada Clinical Trials Group study BR.21. J. Thorac. Oncol. 3(6), 590-598 (2008).

69. Di Maio M, Lama N, Morabito A et al. Clinical assessment of patients with advanced non-small-cell lung cancer eligible for second-line chemotherapy: a prognostic score from individual data of nine randomized trials. Eur. J. Cancer 46(4), 735-743 (2010).

70. Cao W, Li A-W, Ren S-X et al. Efficacy of first-line chemotherapy affects the second-line setting response in patients with advanced non-small cell lung cancer. Asian Pac. J. Cancer Prev. 15(16), 6799-6804 (2014).

71. Hilberg F, Roth GJ, Krssak M et al. BIBF 1120: triple angiokinase inhibitor with sustained receptor blockade and good antitumor efficacy. Cancer Res. 68(12), 4774-4782 (2008). 
72. European Medicines Agency. Ramucirumab summary of product characteristics, (2018). www.ema.europa.eu/documents/product-information/cyramza-epar-product-information_en.pdf

73. European Medicines Agency. Bevacizumab summary of product characteristics, (2018). www.ema.europa.eu/documents/product-information/avastin-epar-product-information_en.pdf

74. European Medicines Agency. Nivolumab summary of product characteristics. (2018). www.ema.europa.eu/documents/product-information/opdivo-epar-product-information_en.pdf

75. Horn L, Spigel DR, Vokes EE et al. Nivolumab versus docetaxel in previously treated patients with advanced non-small-cell lung cancer: two-year outcomes from two randomized, open-label, Phase III trials (CheckMate 017 and CheckMate 057). J. Clin. Oncol. 35(35), 3924-3933 (2017).

76. Inoue T, Tamiya M, Tamiya A et al. Analysis of early death in Japanese patients with advanced non-small-cell lung cancer treated with nivolumab. Clin. Lung Cancer 19(2), e171-e176 (2018).

77. Garde-Noguera J, Martorell PM, De Julián M et al. Predictive and prognostic clinical and pathological factors of nivolumab efficacy in non-small-cell lung cancer patients. Clin. Transl. Oncol. (2018).

78. Kaderbhai CG, Richard C, Fumet JD et al. Response to first-line chemotherapy regimen to predict efficacy of nivolumab in lung cancer. J. Clin. Oncol. 35(15 Suppl.), Abstract 3026 (2017).

79. Nakahama K, Isa S-I, Tamiya A et al. The association between chemotherapy immediately before nivolumab and outcomes thereafter. Anticancer Res. 37(10), 5885-5891 (2017).

80. Herbst RS, Baas P, Kim D-W et al. Pembrolizumab versus docetaxel for previously treated, PD-L1-positive, advanced non-small-cell lung cancer (KEYNOTE-010): a randomized controlled trial. Lancet 387(10027), 1540-1550 (2016).

81. European Medicines Agency. Atezolizumab summary of product characteristics. (2018). www.ema.europa.eu/documents/product-information/tecentriq-epar-product-information_en.pdf

82. Rittmeyer A, Barlesi F, Waterkamp D et al. Atezolizumab versus docetaxel in patients with previously treated non-small-cell lung cancer (OAK): a Phase III, open-label, multicentre randomized controlled trial. Lancet 389(10066), 255-265 (2017).

83. Fadejeva I, Olschewski H, Hrzenjak A. MicroRNAs as regulators of cisplatin-resistance in non-small cell lung carcinomas. Oncotarget 8(70), 115754-115773 (2017).

84. Hamilton G, Rath B. Pharmacogenetics of platinum-based chemotherapy in non-small cell lung cancer: predictive validity of polymorphisms of ERCC1. Expert. Opin. Drug Metab. Toxicol. 14(1), 17-24 (2018).

85. Urquhart G, Kerr KM, Nicolson $\mathrm{M}$ et al. Serpin b3 is associated with poor survival after chemotherapy and is a potential novel predictive biomarker in advanced non-small-cell lung cancer. J. Thorac. Oncol. 8(12), 1502-1509 (2013).

86. Thakur MK, Gadgeel SM. Predictive and prognostic biomarkers in non-small cell lung cancer. Semin. Respir. Crit. Care Med. 37(5), 760-770 (2016).

87. Chaft JE, Sima CS, Ginsberg MS et al. Clinical outcomes with perioperative chemotherapy in sarcomatoid carcinomas of the lung. J. Thorac. Oncol. 7(9), 1400-1405 (2012).

88. Yamakawa H, Takayanagi N, Ishiguro T, Kagiyama N, Shimizu Y, Sugita Y. A favorable response to cisplatin, pemetrexed and bevacizumab in two cases of invasive mucinous adenocarcinoma formerly known as pneumonic-type mucinous bronchioloalveolar carcinoma. Intern. Med. 52(24), 2781-2784 (2013).

89. Hilberg F, Haslinger C, Chesa PG, Adolf GR. Molecular correlates of clinical benefit from antiangiogenic therapy for patients with lung adenocarcinoma: a hypothesis. J. Clin. Oncol. 32(15 Suppl.), Abstract e22080 (2014).

90. Warth A, Cortis J, Soltermann A et al. Tumour cell proliferation (Ki-67) in non-small cell lung cancer: a critical reappraisal of its prognostic role. Br. J. Cancer 111(6), 1222-1229 (2014).

91. Horsman MR, Vaupel P. Pathophysiological basis for the formation of the tumor microenvironment. Front. Oncol. 6, 66 (2016).

92. Eales KL, Hollinshead KER, Tennant DA. Hypoxia and metabolic adaptation of cancer cells. Oncogenesis 5, e190 (2016).

93. Vaupel P, Multhoff G. Accomplices of the hypoxic tumor microenvironment compromising antitumor immunity: adenosine, lactate, acidosis, vascular endothelial growth factor, potassium ions, and phosphatidylserine. Front. Immunol. 8, 1887 (2017).

94. Vaupel P. Hypoxia and aggressive tumor phenotype: implications for therapy and prognosis. Oncologist 13(Suppl. 3), 21-26 (2008).

95. Morabito A. Second-line treatment for advanced NSCLC without actionable mutations: is immunotherapy the 'panacea' for all patients? BMC Med. 16(1), 24 (2018).

96. Bluthgen $\mathrm{M}-\mathrm{V}$, Besse B. Second-line combination therapies in nonsmall cell lung cancer without known driver mutations. Eur. Respir. Rev. 24(138), 582-593 (2015).

97. Tapia G, Diaz-Padilla I. Molecular mechanisms of platinum resistance in ovarian cancer. In: Ovarian Cancer - A Clinical And Translational Update. Diaz-Padilla I (Ed.). IntechOpen, 205-223 (2013). www.intechopen.com/books/ovarian-cancer-a-clinical-and-tra nslational-update/molecular-mechanisms-of-platinum-resistance-in-ovarian-cancer 
98. Reck M, Staal-Van den Brekel AJ, Mellemgaard A et al. A non-interventional biomarker study in patients (pts) with non-small cell lung cancer (NSCLC) of adenocarcinoma histology who are treated with nintedanib according to the approved label (LUME-BioNIS). Ann. Oncol. 28(Suppl. 2), 494 Abstract 139TiP (2017). 
